\title{
Effect of Endovascular Treatment on Headache in Elderly Patients with Unruptured Intracranial Aneurysms
}

\author{
D.-Q. Gu, C.-Z. Duan, X.-F. Li, X.-Y. He, L.-F. Lai, and S.-X. Su
}

\begin{abstract}
BACKGROUND AND PURPOSE: The incidence of unruptured intracranial aneurysms is increasing in the elderly population as life expectancy increases, and patients often present with headache. The goal of this study was to determine the effect of endovascular treatment on headache and identify factors associated with headache outcome in elderly patients with unruptured intracranial aneurysms.
\end{abstract}

MATERIALS AND METHODS: A retrospective study was conducted for elderly patients ( $\geq 65$ years old) being treated for unruptured intracranial aneurysms. Headache assessment was performed by a quantitative 11-point headache scale in all patients before and after endovascular treatment. Factors associated with headache outcome were investigated by univariate analyses.

RESULTS: A total of 72 patients (mean age, 70.0 years; age range, 65-80 years; 41 women) fulfilled the inclusion criteria. There were 52 patients (72.2\%) who presented with preoperative headache (headache score $\geq 1)$. Among them, 40 (76.9\%) reported that headache score had an improvement according to their self-reported quantitative headache score after endovascular treatment. The average headache score was 5.63 preoperatively vs 2.50 postoperatively $(P=.000)$. Twenty patients $(27.8 \%)$ had no headache before treatment (headache score $=0$ ), of whom $2(10.0 \%)$ reported new onset of headache postoperatively. Only a preoperative headache score was associated with treatment outcome of headache, and a higher headache score predicted a lack of headache relief after endovascular treatment $(P=.003)$.

CONCLUSIONS: Endovascular coiling of unruptured intracranial aneurysms resulted in relief of headache in most of the elderly patients. Preoperative headache score was the only statistically significant predictor of headache outcome.

l: tracranial aneurysms are fairly common in the general population. It is estimated that between $0.4 \%$ and $6 \%$ of people may harbor a cerebral aneurysm. ${ }^{1-4}$ Because of noninvasive imaging techniques, including CT, MR imaging, CT angiography, and MR angiography, unruptured intracranial aneurysms are increasingly diagnosed. Headache is a frequent symptom in patients with unruptured aneurysms, often leading to the diagnosis of aneurysm. The literature has revealed that headache was the presenting symptom of unruptured intracranial aneurysms in approximately one-third of patients. ${ }^{5}$ The severity and frequency of headache may change in different ways, including improvement, aggravation, and new onset after endovascular treatment of intracranial aneurysms. $^{6}$

With a globally aging population, the number of elderly pa-

Received May 31, 2012; accepted after revision August 20.

From the Department of Neurosurgery, Neurosurgery Institute, Key Laboratory on Brain Function Repair and Regeneration of Guangdong, Zhujiang Hospital, Southern Medical University, Guangzhou, Guangdong, China.

Please address correspondence to Chuan-Zhi Duan, Department of Neurosurgery, Zhujiang Hospital, Southern Medical University, 253\# Industry Rd, 510282, Guangzhou, Guangdong, China; e-mail: chuanzhiduan@126.com

http://dx.doi.org/10.3174/ajnr.A3353 tients with intracranial aneurysms increases rapidly. Because of possible devastating consequences of aneurysm rupture and low morbidity and mortality rates of endovascular treatment, more and more elderly patients with unruptured intracranial aneurysms have been treated endovascularly. To date, the effect of endovascular treatment on headache in elderly patients with unruptured intracranial aneurysms is not very clear. The objective of this study was to determine the effect of endovascular treatment on headache and identify factors associated with different headache outcomes in elderly patients with unruptured intracranial aneurysms.

\section{MATERIALS AND METHODS Patients}

Endovascular treatment is the first therapeutic option for both ruptured and unruptured intracranial aneurysms at our institution. Approval was obtained from the institutional review board to conduct this study. Included in the study were patients 65 years and older with unruptured intracranial aneurysms treated by endovascular coiling between May 2007 and April 2011. Patients with the following conditions were excluded from this study: 1) other brain lesions, potentially contributing to the headache, 


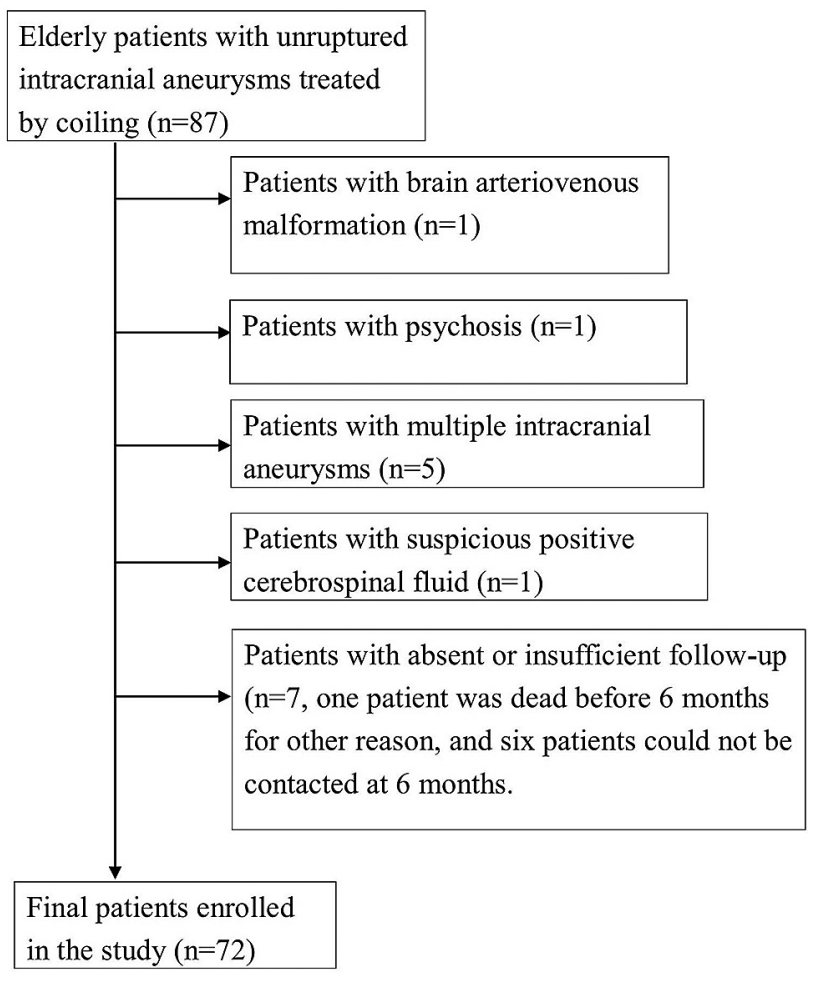

Flow diagram of patients being selected.

such as cerebral infarction, cavernous angioma, arteriovenous malformation, intracerebral hemorrhage, trigeminal neuralgia, Parkinson disease, or brain tumors; 2 ) other comorbid diseases such as psychosis and hepatic encephalopathy; 3 ) multiple intracranial aneurysms; 4) a suspicious positive finding in CSF study; and 5) absent or insufficient follow-up. The cases that were included in our study were identified from a retrospective chart review. The Figure demonstrates a flow diagram of patients being selected.

\section{Endovascular Procedures}

All patients underwent endovascular treatment under general anesthesia. A heparin bolus of 5000 IU was routinely administered as soon as the $6 \mathrm{~F}$ introducer sheath was placed in the common femoral artery. Throughout the procedure, the activated clotting time was kept at 2 to 3 times above the normal value with a continuous heparin infusion of 2500 to $3000 \mathrm{IU} / \mathrm{h}$. No medication that specifically treats headache was used after coiling of the aneurysm.

A $6 \mathrm{~F}$ guiding catheter was selectively placed in the artery supplying the aneurysm. Once the optimal angiographic projection was defined, aneurysm catheterization was performed by advancing a microcatheter over a microguidewire. Coils were placed through the microcatheter into the aneurysm and were detached under fluoroscopy. This process was repeated until the aneurysm was occluded as densely as possible. Several types of coils were used (GDC Serials and Matrix coils, Boston Scientific, Natick, Massachusetts; or EDC Serials, ev3 Neurovascular, Irvine, California) and variable degrees of stiffness are available, including standard, soft, and ultrasoft coils as well as 3D coils. In the case of wide-neck intracranial aneurysms in which coiling only was dif- ficult, stents were positioned across the neck of the aneurysms before coiling. Three types of stents were used, including the Neuroform stent (Neuroform2 or Neuroform3, Boston Scientific), the LEO stent (Balt Extrusion, Montmorency, France), and the Enterprise stent (Codman \& Shurtleff, Raynham, Massachusetts). Some patients were treated with the balloon remodeling technique.

\section{Data Collection}

We obtained patient data by consulting the computer data base of our institution. Some patients were also interviewed over the telephone to evaluate the clinical outcome.

Using a validated, quantitative 11-point headache scale, ${ }^{7}$ we performed a standardized headache assessment on every patient initially presenting for consultation. The same headache assessment was performed at 6 months after endovascular treatment. It is a standard protocol to conduct such a headache assessment before and after treatment in all patients with unruptured intracranial aneurysms at our institution. The quantitative, interval headache score was a scale ranging from 0 to 10 , with 0 being defined as "no headache at all" and 10 being defined as "the worst headache you have ever experienced." The patients self-reported the headache score on the basis of this 11-point scale. A change of headache status after treatment was classified as improvement (reduction in headache score), aggravation (increase in headache score), and no change.

The following patient information was also recorded and used for the present analysis: 1) patient demographics; 2) characteristics of the aneurysm, including its location and size; 3 ) modalities of treatment, classified as standard treatment with coils, stentassisted coiling, and the remodeling technique; 4) complications related to endovascular treatment, including thromboembolic events and intraoperative rupture; 5) side of the headache, including ipsilateral headache to the aneurysm, contralateral headache to the aneurysm, and bilateral headache.

\section{Statistical Analysis}

Frequencies and percentages were calculated for categoric variables, and means and range were calculated for continuous variables. Categoric data were analyzed by use of the Fisher exact test or the Pearson $\chi^{2}$ test, as appropriate. Analyses of continuous data with nonnormal distribution were performed with the Wilcoxon 2 -sample test. The level of statistical significance used was $P<.05$ for the entire study. Statistical analysis was performed with the SPSS statistical package (SPSS 13.0; SPSS, Chicago, Illinois).

\section{RESULTS}

\section{Patients and Aneurysms}

A total of 72 patients (mean age, 70.0 years; age range, $65-80$ years; 41 women) with 72 unruptured intracranial aneurysms fulfilled the inclusion criteria. There were 52 patients $(72.2 \%)$ who presented with preoperative headache (headache score $\geq 1$ ). Headache-related symptoms included nausea $(n=32)$, vomiting $(n=12)$, photophobia $(n=22)$, and phonophobia $(n=20)$. Twenty patients $(27.8 \%)$ had no headache before treatment (headache score $=0$ ).

Fifty-five aneurysms (76.4\%) were located in the anterior cir- 
Table 1: Clinical and angiographic characteristics

\begin{tabular}{lc}
\hline No. of patients & 72 \\
No. of aneurysms & 72 \\
Sex (\%) & \\
$\quad$ Female & $41(56.9)$ \\
$\quad$ Male & $31(43.1)$ \\
Age (y) & \\
$\quad$ Mean & 70.0 \\
$\quad$ Range & $65-80$ \\
Aneurysm location (\%) & \\
ICA & $27(37.5)$ \\
ACA, AcomA & $16(22.2)$ \\
MCA & $12(16.7)$ \\
$\quad$ VB & $17(23.6)$ \\
Aneurysm size (\%) & \\
$\quad<10$ mm & $42(58.3)$ \\
$\quad \geq 10$ mm & $30(41.7)$ \\
Modalities of treatment (\%) & \\
Coiling alone & $51(70.8)$ \\
Stent-assisted coiling & $12(16.7)$ \\
Remodeling & $9(12.5)$ \\
\hline
\end{tabular}

ACA indicates anterior cerebral artery; AcomA, anterior communicating artery; MCA, middle cerebral artery; ICA, internal carotid artery; VB, vertebrobasilar system.

culation and $17(23.6 \%)$ in the posterior circulation. There were $42(58.3 \%)$ small $(<10 \mathrm{~mm})$, and $30(41.7 \%)$ large $(\geq 10 \mathrm{~mm})$ aneurysms. Univariate analysis found no statistically significant correlation of preoperative headache with the location (anterior circulation vs posterior circulation, $P=.764)$ and size $(<10 \mathrm{~mm}$ vs $\geq 10 \mathrm{~mm} ; P=.859$ ) of the aneurysms. Clinical and angiographic characteristics are summarized in Table 1.

\section{Modalities of Endovascular Treatment}

Of the 72 patients, $51(70.8 \%)$ were treated with coils alone, 9 $(12.5 \%)$ were treated with the balloon-remodeling technique, and $12(16.7 \%)$ were treated with stent-assisted coiling (Table 1).

\section{Complications Related to Endovascular Treatment}

Two patients $(2.8 \%)$ had coiling-related complications. Intraoperative rupture occurred in 1 patient $(1.4 \%)$. Bleeding stopped with further coiling, and this patient did not present with neurologic deficits after treatment. Another patient (1.4\%) had thromboembolic events during the procedure. After administration of anticoagulant, the clot shrunk without any clinical complications after coiling.

\section{Change of Headache after Treatment}

Of the 52 patients with preoperative headache, 2 (3.8\%) reported aggravation of the headache after coiling according to preoperative and postoperative headache scores. Ten patients $(19.2 \%)$ stated no change in their headache. Forty patients $(76.9 \%)$ re- cular treatment, headache scores of the patients were 4 and 3 , respectively.

\section{Effect of Clinical Factors on Relief of Headache}

Many prognostic variables were analyzed including age, sex, size and location of the aneurysm, morphologic features of the aneurysm (complex vs single-domed), treatment modalities, side of the headache, and preoperative severity of the headache. Only the preoperative headache score was associated with treatment outcome of headache, and a higher headache score predicted a lack of headache relief after endovascular treatment $(P=.003$; Table 3$)$.

\section{DISCUSSION}

This study describes our experience in the treatment of elderly patients ( $\geq 65$ years old) with unruptured intracranial aneurysms at our institution for the past few years. We found that endovascular coiling of unruptured intracranial aneurysms resulted in relief of headache in most elderly patients with preoperative headache, and that patients with more severe headache were less likely to improve after endovascular treatment.

Increased detection of unruptured intracranial aneurysms is a consequence of development and more common use of advanced diagnostic cerebrovascular imaging techniques such as MR imaging or MR angiography, 3D CT angiography, and digital subtraction angiography. ${ }^{8,9}$ With a globally aging population, the number of elderly patients with unruptured intracranial aneurysms increases rapidly. Headache is one of the most common symptoms leading to the diagnosis of an unruptured intracranial aneurysm. ${ }^{5}$

Since their introduction, endovascular technologies have evolved into one of the most important management options for intracranial aneurysms, and the techniques have been shown to be generally safe and effective. ${ }^{10-12}$ Technologic advances have "pushed the envelope" to make the techniques more durable and more applicable to complex types of aneurysms. ${ }^{13-15}$ Regarding unruptured intracranial aneurysms, many previous investigations ${ }^{16-20}$ have focused on the risks for rupture and hemorrhage while the aneurysms were left untreated, as well as the burden of risk that is associated with treatment modalities (surgical vs endovascular treatment) when the aneurysms are to be treated. ${ }^{21,22}$ Several studies ${ }^{6,23-25}$ have also mentioned the effect of treatment of unruptured intracranial aneurysms on headache. However, literature specifically evaluating headache outcome after endovascular treatment of elderly patients with unruptured aneurysms is rare.

It has been recognized previously that a high proportion of ported that their headache was relieved.

Overall, headache scores had an improvement in self-reported quantitative headache score after endovascular treatment for the 52 patients. The average headache score was 5.63 preoperatively vs 2.50 postoperatively $(P=.000$; Table 2$)$.

Of the 20 patients without preoperative headache, $2(10.0 \%)$ reported new onset of headache postoperatively. After endovas-
Table 2: Evolution of headache after endovascular treatment

\begin{tabular}{lcc}
\hline & $\begin{array}{c}\text { Patients with } \\
\text { Preoperative Headache } \\
(\boldsymbol{n}=\mathbf{5 2})\end{array}$ & $\begin{array}{c}\text { Patients without } \\
\text { Preoperative Headache } \\
(\boldsymbol{n}=\mathbf{2 0})\end{array}$ \\
\hline Preoperative headache score (mean) & 5.63 & $\mathrm{~N} / \mathrm{A}$ \\
Postoperative headache score (mean) & 2.50 & $\mathrm{~N} / \mathrm{A}$ \\
Headache relief (\%) & $40(76.9)$ & $\mathrm{N} / \mathrm{A}$ \\
Headache worsening or new onset (\%) & $2(3.8)$ & $2(10.0)$ \\
No change in headache (\%) & $10(19.2)$ & $18(90.0)$ \\
\hline
\end{tabular}

Note:-Postoperative headache score is statistically different than preoperative headache score $(P=.000)$. N/A indicates not applicable. 
Table 3: Univariate statistical analysis of factors associated with headache outcome among patients with preoperative headache

\begin{tabular}{|c|c|c|c|}
\hline & $\begin{array}{l}\text { Headache Relief } \\
\qquad(n=40)\end{array}$ & $\begin{array}{l}\text { No Headache Relief } \\
\qquad(n=12)\end{array}$ & $P$ value \\
\hline Mean age (y) & 69.2 & 71.1 & .212 \\
\hline Female sex (\%) & $24(60.0)$ & $5(41.7)$ & .262 \\
\hline Aneurysm location ${ }^{\mathrm{a}}$ in anterior circulation (\%) & $31(77.5)$ & $8(66.7)$ & .466 \\
\hline Aneurysm size $<10 \mathrm{~mm}(\%)$ & $24(60.0)$ & $6(50.0)$ & .539 \\
\hline Single-domed morphologic feature ${ }^{\mathrm{b}}$ of aneurysm (\%) & $35(87.5)$ & $9(75.0)$ & .366 \\
\hline \multicolumn{4}{|l|}{ Modalities of treatment (\%) } \\
\hline Coiling alone & $28(70.0)$ & $6(50.0)$ & .300 \\
\hline Stent-assisted coiling & $7(17.5)$ & $4(33.3)$ & .253 \\
\hline Remodeling & $5(12.5)$ & $2(16.7)$ & .656 \\
\hline \multicolumn{4}{|l|}{ Side of headache to aneurysm (\%) } \\
\hline Ipsilateral & $12(30.0)$ & $4(33.3)$ & 1.000 \\
\hline Contralateral & $10(25.0)$ & $5(41.7)$ & .293 \\
\hline Bilateral & $18(45.0)$ & $3(25.0)$ & .318 \\
\hline Preoperative headache score (mean) & 5.28 & $6.83^{\prime}$ & .003 \\
\hline
\end{tabular}

Location of aneurysm is classified as anterior and posterior circulation.

${ }^{b}$ Morphologic features of aneurysm are classified as complex and single-domed.

patients with unruptured intracranial aneurysms have headache. ${ }^{5,26,27}$ The mechanisms that cause headache associated with an unruptured aneurysm include local thrombosis, localized meningeal inflammation, expansion of the aneurysm, and bleeding within the vascular wall. ${ }^{27}$ The explanation for the change in headache status after aneurysm treatment is not clear. Coil embolization may reduce the pulsatile expansion of the aneurysm sac, which leads to relief of the headache. ${ }^{6}$ In addition, the development of a fibrotic response within the embolized aneurysm may result in retraction of the aneurysms. ${ }^{6}$ Worsening or development of new headache may be attributed to increase in the size of the aneurysm after placement of coils. ${ }^{28}$ Another explanation for worsening of headache immediately after embolization may be the inflammatory and thrombotic response invoked by coils within the aneurysm sac. ${ }^{29}$ Our study suggested that elderly patients with unruptured intracranial aneurysms experience headache frequently. Among the 72 patients in our series, 52 (72.2\%) had headache before endovascular treatment. Our study also revealed a relatively high rate $(76.9 \%)$ of headache relief among elderly patients with preoperative headache.

Experiences regarding new headache after aneurysm embolization have been described in previous studies. ${ }^{6,30}$ In a study by Qureshi et al, ${ }^{6} 5$ of 15 patients without headache before embolization reported onset of mild $(n=4)$ or severe $(n=1)$ headache after treatment. Hwang et $\mathrm{al}^{30}$ reported that 50 of 90 patients without a history of headache within 1 month before treatment experienced a headache at 7.9 hours, on average, after coiling, and all headaches resolved within an average of 73.0 hours. Compared with published series, our study revealed a relatively low rate $(10.0 \%)$ of new headache among patients without preoperative headache after treatment. The exact reason for the difference is not yet well known. Different clinical characteristics, coil materials, and follow-up time may partly contribute to the difference.

Univariate exploratory analyses identified several possible predictors for headache resolution after endovascular treatment. The preoperative headache score was the only statistically significant predictor of treatment outcome of headache, and a higher headache score predicted a lack of headache relief after endovascular treatment $(P=.003)$. The mechanism is not clear. Other factors including age, sex, size and location of the aneurysm, mor- phologic features of the aneurysm (complex vs single-domed), treatment modalities, and side of the headache did not show associations with headache outcome after treatment. These associations should be further assessed in future larger studies that are powered for multivariate analyses.

To date, the optimal management of unruptured intracranial aneurysms remains controversial in the elderly population, especially small, unruptured aneurysms. Literature specifically evaluating endovascular treatment of elderly patients with unruptured aneurysms is rare. The decision to treat or to observe an identified unruptured aneurysm in an elderly patient requires an honest assessment of treatment-related risks, a careful estimation of the involved patient's life expectancy, and a precise knowledge of the natural history of the disease process. Many physicians have not recommended active treatment of unruptured intracranial aneurysms for older patients because of their relatively short life expectancy, potential hazards related to treatment, and relatively low rate of aneurysm rupture. However, many elderly patients with unruptured intracranial aneurysms complain of headache and seek treatment for pain relief. Our study revealed a relatively high rate of headache relief among elderly patients with preoperative headache. Moreover, our series demonstrated that endovascular coiling of unruptured intracranial aneurysms in elderly patients is safe, with a low rate $(2.8 \%)$ of complications. The results presented in our study may help to better understand endovascular treatment of unruptured intracranial aneurysms in elderly patients. Larger, prospective series are required to adequately assess the effect of treatment and draw definitive conclusions.

To our knowledge, this study is the first analysis of headache outcome after endovascular treatment of unruptured intracranial aneurysms in elderly patients. However, our study had several limitations. First, the number of cases of patients may not have been large enough to draw any definitive conclusions. Second, patients excluded from this study because of absent or insufficient follow-up may have led to a source of unintentional selection bias that could not be overcome with this study design. Third, the relief from headache that we found in this study may have been exaggerated because of a possible placebo effect of endovascular treatment. 


\section{CONCLUSIONS}

Endovascular coiling of unruptured intracranial aneurysms resulted in relief of headache in most of these elderly patients. The preoperative headache score was the only statistically significant predictor of headache outcome. Further larger studies are required to adequately assess the effect of endovascular treatment on headache in the elderly population.

\section{REFERENCES}

1. Rinkel GJ, Djibuti M, Algra A, et al. Prevalence and risk of rupture of intracranial aneurysms: a systematic review. Stroke 1998;29:251-56

2. Schievink WI. Intracranial aneurysms. $N$ Engl J Med 1997;336: $28-40$

3. Horikoshi T, Akiyama I, Yamagata Z, et al. Retrospective analysis of the prevalence of asymptomatic cerebral aneurysm in 4518 patients undergoing magnetic resonance angiography-when does cerebral aneurysm develop? Neurol Med Chir (Tokyo) 2002;42:105-12; discussion 113

4. Winn HR, Jane JA Sr, Taylor J, et al. Prevalence of asymptomatic incidental aneurysms: review of 4568 arteriograms. J Neurosurg 2002;96:43-49

5. Unruptured intracranial aneurysms - risk of rupture and risks of surgical intervention. International Study of Unruptured Intracranial Aneurysms Investigators. N Engl J Med 1998;339:1725-33

6. Qureshi AI, Suri MF, Kim SH, et al. Effect of endovascular treatment on headaches in patients with unruptured intracranial aneurysms. Headache 2003;43:1090-96

7. Kwong WJ, Pathak DS. Validation of the eleven-point pain scale in the measurement of migraine headache pain. Cephalalgia 2007;27: $336-42$

8. Pechlivanis I, Schmieder K, Scholz M, et al. 3-dimensional computed tomographic angiography for use of surgery planning in patients with intracranial aneurysms. Acta Neurochir (Wien) 2005;147:104553; discussion 1053

9. Wiebers DO, Piepgras DG, Meyer FB, et al. Pathogenesis, natural history, and treatment of unruptured intracranial aneurysms. Mayo Clin Proc 2004;79:1572-83

10. Gonzalez N, Murayama Y, Nien YL, et al. Treatment of unruptured aneurysms with GDCs: clinical experience with 247 aneurysms. AJNR Am J Neuroradiol 2004;25:577-83

11. Guglielmi G, Viñuela F, Duckwiler G, et al. Endovascular treatment of posterior circulation aneurysms by electrothrombosis using electrically detachable coils. J Neurosurg 1992;77:515-24

12. Murayama Y, Nien YL, Duckwiler G, et al. Guglielmi detachable coil embolization of cerebral aneurysms: 11 years' experience. J Neurosurg 2003;98:959-66

13. Benitez RP, Silva MT, Klem J, et al. Endovascular occlusion of widenecked aneurysms with a new intracranial microstent (Neuroform) and detachable coils. Neurosurgery 2004;54:1359-67; discussion 1368

14. Lylyk P, Ferrario A, Pasbón B, et al. Buenos Aires experience with the Neuroform self-expanding stent for the treatment of intracranial aneurysms. J Neurosurg 2005;102:235-41

15. Taschner CA, Leclerc X, Rachdi H, et al. Matrix detachable coils for the endovascular treatment of intracranial aneurysms: analysis of early angiographic and clinical outcomes. Stroke 2005;36:2176-80

16. Matsumoto K, Akagi K, Abekura M, et al. Investigation of the surgically treated and untreated unruptured cerebral aneurysms of the anterior circulation. Surg Neurol 2003;60:516-22; discussion 522-23

17. Morita A, Fujiwara S, Hashi K, et al. Risk of rupture associated with intact cerebral aneurysms in the Japanese population: a systematic review of the literature from Japan. J Neurosurg 2005;102:601-66

18. Tsutsumi K, Ueki K, Morita A, et al. Risk of rupture from incidental cerebral aneurysms. J Neurosurg 2000;93:550-53

19. Wiebers DO. The risk of rupture of unruptured cerebral aneurysms in the Japanese population: a systematic review of the literature from Japan by Morita, et al. J Neurosurg 2005;102:597; discussion 597-98

20. Yasui N, Suzuki A, Nishimura H, et al. Long-term follow-up study of unruptured intracranial aneurysms. Neurosurgery 1997;40:115559; discussion 1159-60

21. Niskanen M, Koivisto T, Rinne J, et al. Complications and postoperative care in patients undergoing treatment for unruptured intracranial aneurysms. J Neurosurg Anesthesiol 2005;17:100-05

22. Vindlacheruvu RR, Mendelow AD, Mitchell P. Risk-benefit analysis of the treatment of unruptured intracranial aneurysms. J Neurol Neurosurg Psychiatry 2005;76:234-39

23. Kong DS, Hong SC, Jung YJ, et al. Improvement of chronic headache after treatment of unruptured intracranial aneurysms. Headache 2007;47:693-97

24. Schwedt TJ, Gereau RW, Frey K, et al. Headache outcomes following treatment of unruptured intracranial aneurysms: A prospective analysis. Cephalalgia 2011;31:1082-89

25. Choxi AA, Durrani AK, Mericle RA. Both surgical clipping and endovascular embolization of unruptured intracranial aneurysms are associated with long-term improvement in self-reported quantitative headache scores. Neurosurgery 2011;69:128-33; discussion 133-34

26. Deruty R, Pelissou-Guyotat I, Mottolese C, et al. Management of unruptured cerebral aneurysms. Neurol Res 1996;18:39-44

27. Raps EC, Rogers JD, Galetta SL, et al. The clinical spectrum of unruptured intracranial aneurysms. Arch Neurol 1993;50:265-68

28. Thornton J, Dovey Z, Alazzaz A, et al. Surgery following endovascular coiling of intracranial aneurysms. Surg Neurol 2000;54:352-60

29. Murayama Y, Vinuela F, Tateshima S, et al. Bioabsorbable polymeric material coil for embolization of intracranial aneurysms: a preliminary experimental study. J Neurosurg 2001;94:454-63

30. Hwang G, Jeong EA, Sohn JH, et al. The characteristics and risk factors of headache development after the coil embolization of an unruptured aneurysm. AJNR Am J Neuroradiol 2012;33:1676-78 